\title{
Psychophysical scaling of smiling faces
}

\author{
HIROSHI HOJO' \\ Department of Educational Psychology, School of Education, Waseda University, Shinjuku-ku, Tokyo 160
}

\begin{abstract}
Sixteen schematic faces with two defining properties of eyes and lips drawn by arcs with varying curvatures were rank ordered by 37 university students according to their perceived intensities of smiling. The data were analyzed by a nonmetric maximum likelihood scaling method for directional rank order data. The analysis revealed (a) that the unconstrained model assuming no psychophysical relationships between the two curvatures of eyes and lips and the judged intensity of smiling fits the data better than a psychophysical function having a linear combination of the two curvatures as an independent variable, or than an additive function of two psychological effects clicited by eyes and lips, respectively; (b) but that the psychophysical function with a term of interaction effects between the two properties is the most appropriate model of the perceived intensity of smiling; (c) that eyes are interpreted twice as important as lips in perception of intensity of smiling of faces.
\end{abstract}

Key words: psychophysical scaling, maximum likelihood estimation, directional rank orders, facial expressions, Akaike's information criterion (AIC) statistic.

Inukai, Shinohara, and Nakamura (1977), Inukai (1981), and Takane and Carroll (1981) have analyzed similarities among 17 schematic faces whose defining properties are eyes and lips depicted by arcs with varying curvatures. They have found that the curvatures of eyes and lips are closely related to the perception of facial expressions. For example, faces with convex eyes and concave lips have been found to give "positive feelings" (Inukai, 1981). However, they did not go into more specific analyses of relations between the perception of facial expressions and the two curvatures themselves.

The question to be posed in this paper is how the perception of intensity of smiling of a face is related to the two curvatures of eyes and lips. More concretely, we will ask if any particular functional relationships can be assumed between the subjectively experienced intensity of smiling of a face and the two curvatures of eyes and lips. The question will be also discussed whether the perceived intensity

1 The author wishes to express his thanks to Professor Tatsuro Makino of Waseda University for his interest and valuable advice during the course of this work. of smiling can be represented as a simple addition of two psychological effects elicited by eyes and lips, respectively.

Our general approach is a "parametric approach" to nonmentric scaling following the terminology suggested by Takane and Carroll (1981). (For comprehensive references on this approach see, for example, Takane, 1978, Takane, 1981, and Takane and Sergent, 1983.)

\section{The Data}

Stimuli were a set of 16 schematic faces (see Fig. 1), which were constructed by combining four levels each of curvatures of eyes and lips. The major and minor axes of a face were 6.8 and $5.1(\mathrm{~cm})$ long, respectively. In eyes and lips four curvature levels, $0.36,0.71,1.11$, and 1.43, were used. All eyes were concave and all lips convex, but we used absolute values to indicate curvatures of both eyes and lips. Thirty-seven university students judged the degrees of smiling of those 16 faces by the directional ranking method (Hojo, 1985; Takane \& Carroll, 1981). The subject was presented with 16 faces at a time, and was first instructed to choose 
the face judged to be most intensely smiling among the 16 faces, then to choose the next most intensely smiling face among the 15 remaining faces, and so on until a complete ranking was obtained among the 16 stimuli.

\section{The Models}

As candidates for a model of the judged intensity of smiling of a face we choose the following four models:

1. The unconstrained model (UCM). UCM assumes no particular functional relationships between the perceived intensity of smiling and the two curvatures of eyes and lips. Usually, UCM is represented as a vector of scale values assigned to each stimulus, such as

$$
\begin{aligned}
& \boldsymbol{s}=\left(s_{11}, \ldots, s_{i j}, \ldots, s_{N_{N^{N}} N_{L}}\right) \\
& \quad\left(i=1, \ldots, \mathcal{N}_{E} ; j=1, \ldots, \mathcal{N}_{L}\right)
\end{aligned}
$$

where $s_{i j}$ is the scale value representing the perceived intensity of smiling of the stimulus with the $i^{\text {th }}$ eye curvature level and the $j^{\text {th }}$ lip curvature level (This stimulus will be simply designated as stimulus $(i, j)$ in what follows), and $\mathcal{N}_{E}$ and $\mathcal{N}_{L}$ are the numbers of levels of eye curvature and lip curvature, respectively $\left(\mathcal{N}_{E}=\mathcal{N}_{L}=4\right.$ in the present case). Note that we may assume that $s_{i f}=s_{k l}$ for any $i, j, k$, and $l$ if they happen to be close enough. The number of distinct scale values will be represented by $\mathcal{N}_{s}$.

2. The simple additive model $(S A M)$. SAM assumes

$$
s_{i j}=E_{i}+L_{j}+C,
$$

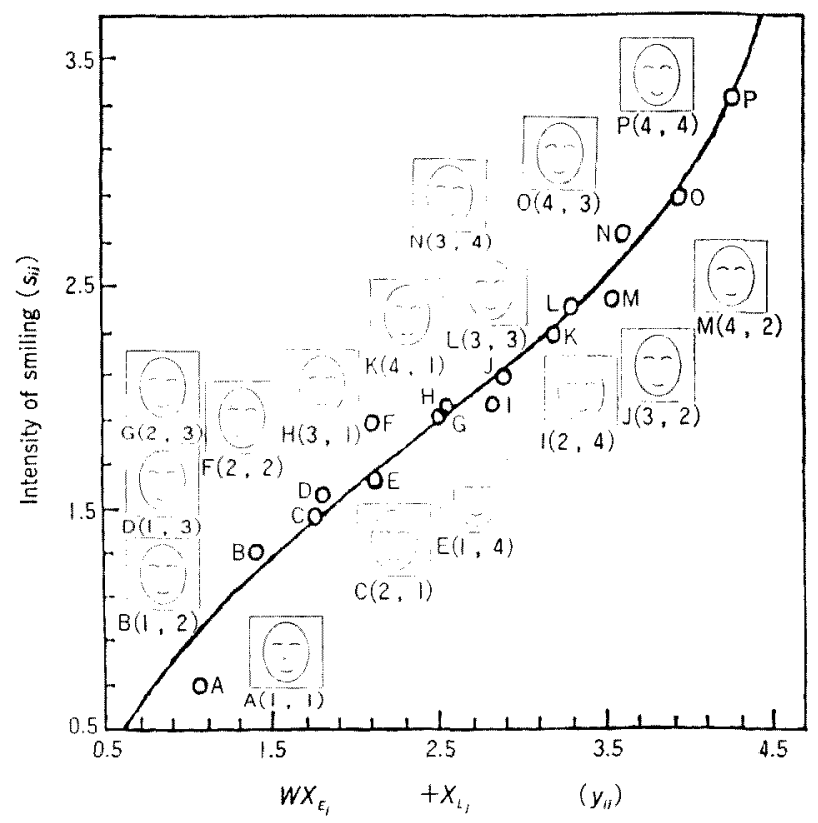

Fig. 1. The perceived smiling intensities of 16 schematic faces estimated from QFM are plotted as a function of physical measure $y_{i j}$. The curve in the graph represents the predictions from PFM in this QFM. A pair of figures in each parenthesis are the levels of eye curvature and lip curvature in the face.

$$
\begin{aligned}
& \text { QFM: } s_{i j}=-\frac{1}{A} \ln \left(\frac{B}{y_{i j}}-1\right)+C+c_{i j} \\
& A=1.46, B=4.72, C=1.80 \\
& c_{11}=-.252, c_{22}=.221, c_{24}=c_{42}=-.120 \\
& c_{34}=c_{12}=c_{13}=.096, c_{31}=c_{21}=c_{39}=c_{23}=.027 \\
& c_{43}=c_{14}=c_{41}=c_{44}=c_{32}=-.037
\end{aligned}
$$


where $E_{i}$ and $L_{j}$ denote the additive effects of the $i^{\text {th }}$ curvature level of eyes and the $j^{\text {th }}$ curvature level of lips, respectively, $C$ is a constant. (See Takane, 1982, for a more extensive discussion of SAM.)

3. The psychophysical function model $(P F, M)$. The PFM proposed hese is an inverse logistic function

$$
s_{i j}=-\frac{1}{A} \ln \left(\frac{B}{y_{i j}}-1\right)+C,
$$

where

$$
y_{1}=a x_{E_{i}}+x_{i_{j}},
$$

and where $r_{i j}$ is the physical measure of stimulus $(i, j), w$ is a weight attached to eyes ( 1 is attached to lips a priori), $x_{E_{t}}$ is the physical value of the $i^{\text {th }}$ curvature level of the eyes, $x_{L}$, is that for the lip curvature, and $A, B$, and $C$ are constants. Model (3) states that changes in the lower and upper ranges of $y_{i j}$ are steep, and that in the middle ranges of $y_{i f}$ the slope is less steep (Fig. 1). We choose (3) rather than a linear function model $\left(s_{i j}=A y_{i j}+C\right)$ because the former was found the better than the latter by pilot studies.

4. The quasi-psychophysical function model (QFM). We present another type of UCM called the quasi-psychophysical function model. In this model smiling intensity parameter $s_{i j}$ given in UCM is restricted in such a way that

$$
s_{i j}=-\frac{1}{A} \ln \left(\frac{B}{y_{i j}}-1\right)+C+c_{i j},
$$

where the first two terms in the right side correspond to PFM defined by (3) and $c_{i j}$ is a correction parameter which represents the fraction of intensity that cannot be accounted for by PFM alone. Although $c_{i j}$ is specific to each stimulus $(i, j)$, we have to impose a restriction $c_{i j}=c_{k l}$ for any $i, j, k$, and $l$ if they are close enough in order to avoid overparametrization. One drawback in this model is that the correction parameter is empirically meaningless unless it can be interpreted in a concrete way. However, at least we can safely expect that the more PFM in QFM can describe data, the smaller the number of distinct values for this parameter, expressed by $\mathcal{N}_{c}$, tends to become. (See Hojo, 1985, for a fuller account of UCM, PFM, and QFM. An example of their applications to scaling models will be also seen in Hojo, 1986.)

Now, we assume that the model prediction defined above is error perturbed. The crror perturbed prediction is not directly observed in the data collection method. By obtaining rank orders some bit of information is assumed to get lost through a specific information reduction mechanism which is presumed to underlie the subject's judgmental process. The model of this information reduction mechanism is generally called the response model. The likelihood of observed data is stated in terms of the likelihood of the error perturbed model prediction via the corresponding response model (Takane, 1982). (The above is a brief description of the parametric approach to nonmetric scaling according to Takane, 1982 and Takane and Carroll, 1981.) Takane and Carroll (1981) have developed the response model for directional rank order data in the framework of multidimensional scaling. A unidimensional version of this model, which can easily be formulated according to Takane and Garroll (1981) (see Hojo, 1985; note that $v$ in (11) in Hojo, 1985, must be replaced by $-v$ in the present case), is used in this analysis, although we do not give its formal expressions in this paper. The joint likelihood for the entire set of observations, $L$, can be stated by means of this response model. The log of $L$ is maximized with respect to parameters in the models by a quasi-Newton method in the present analysis. A BASIC program of this numerical method is taken from Tone (1981).

In evaluating the goodness of fit of the models we use the AIG statistic (Akaike, 1974) defined by 
Table 1

Summary of the results obtained by fitting the four different models of the perceived intensity of smiling

\begin{tabular}{|c|c|c|c|c|}
\hline \multicolumn{2}{|c|}{ Models } & \multirow{2}{*}{$\begin{array}{l}\text { Equality assumptions on scale values or } \\
\text { correction parameters, and weight } \\
\text { values for PFM and QFM } \\
s_{19}=s_{14}, s_{22}=s_{23}=s_{24}=s_{31}, s_{83}=s_{42}\end{array}$} & \multirow{2}{*}{$\begin{array}{l}n_{\phi} \\
\frac{10}{10}\end{array}$} & \multirow{2}{*}{$\begin{array}{l}\text { AIC } \\
1117.8\end{array}$} \\
\hline \multirow{4}{*}{ UCM } & $\mathcal{N}_{8}=11$ & & & \\
\hline & $N_{s}=12$ & $s_{13}=s_{14}, s_{22}=s_{23}, s_{24}=s_{31}, s_{33}=s_{42}$ & 11 & 1113.8 \\
\hline & $\mathcal{N}_{s}=13$ & $s_{22}=s_{28}, s_{24}=s_{31}, s_{33}=s_{42}$ & 12 & 1114.1 \\
\hline & $\mathcal{N}_{s}=14$ & $s_{22}=s_{23}, s_{24}=s_{31}$ & 13 & 1116.0 \\
\hline SAM & & & 8 & 1213.8 \\
\hline PFM & & $w=1.95$ & 3 & l 175.2 \\
\hline \multirow[t]{5}{*}{ QFM } & $\mathcal{N}_{\mathrm{c}}=6$ & $w=2.00$ & 8 & 1107.0 \\
\hline & & $c_{24}=c_{42}$ & & \\
\hline & & $c_{34}=c_{12}=c_{13}$ & & \\
\hline & & $c_{31}=c_{21}=c_{33}=c_{23}$ & & \\
\hline & & $c_{43}=c_{14}=c_{11}=c_{44}=c_{32}$ & & \\
\hline
\end{tabular}

$$
\operatorname{AIC}(\phi)=-2 \ln L+2 n_{\psi},
$$

where $n_{\psi}$ is the effective number of parameters in model $\psi$. The model associated with a smaller value of AIC is considered the better model. The effective numbers of parameters in the four models (1)-(5) are each calculated by

$n_{U C M}=\mathcal{N}_{S}+1$ (for a dispersion of error perturbed model predictions necessary in the response model) -1 (for arbitrary unit of the scale value) -1 (for arbitrary origin),

$n_{S A M}=\mathcal{N}_{E}+\mathcal{N}_{L}+1$ (for a dispersion parameter) -1 (for arbitrary unit of the additive effects),

$n_{P F M}=1($ for $w)+1($ for $A)+1($ for $B)+1$ (for a dispersion parameter) -1 (for arbitrary unit of the scale value),

$n_{Q F M}=n_{P F M}+\mathcal{N}_{c}-1$ (since one of $c_{i j}$ can be fixed to zero without loss of generality).

(Note that constant $C$ does not appear in the response model. See Takane and Carroll, 1981.)

Results of Application and Discussion

The four models were fitted to the rank order data of 16 smiling faces. (Thirtyseven students were taken as mere replications of a single subject.) The main results of analysis are reported in Table 1. From this it can be seen that SAM and PFM are less descriptive of the data than UCM, and that QFM with six correction parameters is the most appropriate model of the subjectively experienced intensity of smiling. In Fig. 1 is plotted the scale value of each stimulus $(i, j)$ predicted from this QFM as a function of physical measure $y_{i j}$. The curve in the figure is the prediction of PFM used in this QFM. From the plot it will be seen that the scale values of stimuli in the middle ranges of $y_{i f}$, such as stimuli $(2,2),(2,3)(3,1)(2,4)$, $(3,2)$, are close to each other as compared with those in the lower and higher ranges of $y_{i j}$, and that the departure from the curve is clearly observed for both stimuli $(1,1)$ and $(2,2)$.

The above result shows that in addition to PFM, that is, the inverse logistic function of $y_{i j}$, we need six correction terms to obtain good account of the data. Since only the curvatures of eyes and lips are varied in the present stimuli, it is natural to ascribe the correction terms to interaction effects of these variables. Unfortunately, however, no clear systematic behavior of their interactions is found in the 
result. Therefore, this result may suggest that there is another type of nonlinearity in the relation between $s_{i j}$ and $y_{i j}$.

The QFM solution has revealed that the weight attached to eyes is twice as large as that attached to lips, implying that eyes can be interpreted twice as important as lips in perception of smiling faces. This finding is worth noting because in the present model the weight is directly attached to the physical value of each curvature level of eyes or lips, but not to the feature of eye or lip as is often the case with studies on saliency of facial features. According to Shepherd, Davies, and Ellis (1981) the most consistent finding in those studies has been that hair is the most important feature. However, Shepherd et al. (1981) point out that this result only reflects the fact that hair has been the most heterogeneous feature in those experiments. From the point of view of psychophysical scaling taken here the above finding can be ascribed to large physical differences which existed among hair styles of stimuli used. It would be natural that features whose values are considerably varied tend to be more heavily weighted in recognition or similarity judgment experiments. In the present model, on the other hand, since the degree of variations of the values over the four eye curvature levels is exactly the same for the lip curvature levels (note further that the weight is attached to the unit of physical value), the estimate of the weight is free from such biases, permitting a meaningful comparison of importance between the two facial features.

In conclusion, the following three points must be noticed: (a) The perceived intensity of smiling of a face cannot be represented as a simple addition of a contribution from eyes and a contribution from lips. (b) Although UGM fits the data better than SAM or PFM, QFM is found most appropriate among the four models. This means that a relatively large portion of variability is accounted for by PFM, but that it is not good enough to explain all meaningful variations in the data, leaving the problem unsolved of what the six correction parameters really mean. (c) Eyes are twice as important as lips in perception of intensity of smiling of faces.

\section{References}

Akaike, H. 1974 A new look at the statistical model identification. IEEE Transactions on Automatic Control, 19, 716-723.

Hojo, H. 1985 Psychophysical scaling of dot dispersion. Japanese Psychological Research, 27, 110.

Hojo, H. 1986 Psychophysical scaling of similarity of pentagons. Japanese Psychological Research, 28, 11-20.

Inukai, Y. 1981 Analysis of perceptual dimensions of schematic facial expressions via three-way multidimensional scaling. Behaviormetrika, 9, 120.

Inukai, Y., Shinohara, M., \& Nakamura, K. 1977 A multidimensional scaling analysis of schematic facial expressions. Bulletin of Industrial Products Research Institute, 81, 21-30. (In Japanese)

Shepherd, J., Davies, G., \& Ellis, H. 1981 Studies of cue saliency. In G. Davies, H. Ellis \& J. Shepherd (Eds.), Perceiving and remembering faces. New York: Academic Press. Pp. 105-131.

Takane, Y. 1978 A maximum likelihood method for nonmetric multidimensional scaling: I. The case in which all empirical pairwise orderings are independent-theory. Japanese Psychological Research, 20, 7-17.

Takane, Y. 1981 Multidimensional successive categories scaling: A maximum likelihood method. Psychometrika, 46, 9-28.

Takane, Y. 1982 Maximum likelihood additivity analysis. Psychomeirika, 47, 225-241.

Takane, Y., \& Carroll, J. D. 1981 Nonmetric maximum likelihood multidimensional scaling from directional rankings of similarities. Psychometrika, 46, 389-405.

Takane, Y., \& Sergent, J. 1983 Multidimensional scaling models for reaction times and samedifferent judgments. Psychometrika, 48, 393-423.

Tone, K. 1981 BASIC. (Microcomputer programs series 1.) Baifukan. (In Japanese)

(Received Nov. 4, 1984; accepted Sept. 14, 1985) 